\title{
The SNMMI and EANM Practice Guideline for Tele-Nuclear Medicine 2.0*
}

\author{
J. Anthony Parker ${ }^{1}$ (Chair), Paul Christian ${ }^{2}$, Hossein Jadvar ${ }^{3}$, Bernhard Sattler ${ }^{4,5}$, and Jerold W. Wallis ${ }^{6}$ \\ ${ }^{1}$ Beth Israel Deaconess Medical Center, Boston, Massachusetts; ${ }^{2}$ Huntsman Cancer Institute, University of Utah, Salt Lake City, \\ Utah; ${ }^{3}$ University of Southern California Keck School of Medicine, Los Angeles, California; ${ }^{4}$ EANM Physics Committee, Vienna, \\ Austria; ${ }^{5}$ University Hospital Leipzig, Leipzig, Germany; and ${ }^{6}$ Mallinckrodt Institute of Radiology, St. Louis, Missouri
}

\section{PREAMBLE}

The Society of Nuclear Medicine and Molecular Imaging (SNMMI) is an international scientific and professional organization founded in 1954 to promote the science, technology, and practical application of nuclear medicine. Its 18,000 members are physicians, technologists, and scientists specializing in the research and practice of nuclear medicine. In addition to publishing journals, newsletters, and books, the SNMMI also sponsors international meetings and workshops designed to increase the competencies of nuclear medicine practitioners and to promote new advances in the science of nuclear medicine. The European Association of Nuclear Medicine (EANM) is a professional nonprofit medical association that facilitates communication worldwide between individuals pursuing clinical and research excellence in nuclear medicine. The EANM was founded in 1985.

The SNMMI/EANM will periodically define new guidelines for nuclear medicine practice to help advance the science of nuclear medicine and to improve the quality of service to patients. Existing practice guidelines will be reviewed for revision or renewal, as appropriate, on their fifth anniversary or sooner, if indicated.

Each practice guideline, representing a policy statement by the SNMMI/EANM, has undergone a thorough consensus process in which it has been subjected to extensive review. The SNMMI/EANM recognizes that the safe and effective use of diagnostic nuclear medicine imaging requires specific training, skills, and techniques, as described in each document.

\footnotetext{
Received Sep. 27, 2013; revision accepted Sep. 27, 2013.

For correspondence or reprints contact: J. Anthony Parker, Beth Israel

Deaconess Medical Center, 330 Brookline Ave., Boston, MA 02215-5491.

E-mail: j.a.parker@ieee.org

Published online Dec. 27, 2013.

*NOTE: FOR CE CREDIT, YOU CAN ACCESS THIS ACTIVITY THROUGH

THE SNMMI WEB SITE (http://www.snmmi.org/ce_online) THROUGH MARCH 2016.

COPYRIGHT (C 2013 by the Society of Nuclear Medicine and Molecular Imaging, Inc.

DOI: 10.2967/jnmt.113.133231
}

The EANM and SNMMI have written and approved these guidelines to promote the use of nuclear medicine procedures with high quality. These guidelines are intended to assist practitioners in providing appropriate nuclear medicine care for patients. They are not inflexible rules or requirements of practice and are not intended, nor should they be used, to establish a legal standard of care. For these reasons and those set forth below, the SNMMI/ EANM cautions against the use of these guidelines in litigation in which the clinical decisions of a practitioner are called into question.

The ultimate judgment regarding the propriety of any specific procedure or course of action must be made by medical professionals taking into account the unique circumstances of each case. Thus, there is no implication that an approach differing from the guidelines, standing alone, is below the standard of care. To the contrary, a conscientious practitioner may responsibly adopt a course of action different from that set forth in the guidelines when, in the reasonable judgment of the practitioner, such course of action is indicated by the condition of the patient, limitations of available resources, or advances in knowledge or technology subsequent to publication of the guidelines.

The practice of medicine involves not only the science but also the art of dealing with the prevention, diagnosis, alleviation, and treatment of disease. The variety and complexity of human conditions make it impossible to always reach the most appropriate diagnosis or to predict with certainty a particular response to treatment. Therefore, it should be recognized that adherence to these guidelines will not ensure an accurate diagnosis or a successful outcome. All that should be expected is that the practitioner will follow a reasonable course of action based on current knowledge, available resources, and the needs of the patient to deliver effective and safe medical care. The sole purpose of these guidelines is to assist practitioners in achieving this objective.

\section{INTRODUCTION}

Tele-nuclear medicine may improve health care by allowing for more timely interpretation and by facilitating consultation. For example, tele-nuclear medicine may 
enable increased availability of nuclear medicine in underserved areas and may be a useful tool for continuing medical education. PACS (1) are increasingly able to provide a remote workstation environment similar to the on-site environment (client-server concept). This guideline focuses on tele-nuclear medicine separate from the main PACS capabilities - that is, on the remote clients of the PACS.

\section{GOALS}

The goal of this guideline is to assist nuclear medicine practitioners in using tele-nuclear medicine for interpretation and consultation of nuclear medicine studies.

\section{DEFINITIONS}

See also the SNMMI Guideline for General Imaging.

Tele-nuclear medicine refers to nuclear medicine interpretation or consultation at a location distant from where the data are acquired. There is a continuum of separation between the physical location of the acquisition and interpretation, but tele-nuclear medicine is meant to imply that the interpretation is relatively remote as compared with the typical interpretation.

Tele-nuclear medicine equipment is used to implement tele-nuclear medicine. The same equipment may be used both on-site and for tele-nuclear medicine (2,3). This guideline will focus on the special considerations required when nuclear medicine equipment is used at remote locations. Distribution of images in a single imaging center falls into the realm of PACS and is not the major focus of this document.

\section{COMMON CLINICAL INDICATIONS}

Common indications for tele-nuclear medicine include, but are not limited to, interpretation of routine studies at a remote location, interpretation of emergency studies in an on-call setting, and consultation.

\section{QUALIFICATIONS AND RESPONSIBILITIES OF PERSONNEL (IN THE UNITED STATES)}

See SNMMI Guideline for General Imaging.

\section{PROCEDURE/SPECIFICATIONS OF THE EXAMINATION}

See also the SNMMI Guideline for General Imaging.

\section{A. Types of tele-nuclear medicine systems}

Tele-nuclear medicine can be implemented as a nuclear medicine-only system, as a part of a tele-radiology system, or as part of another tele-imaging system. In the latter 2 cases, nuclear medicine-specific capabilities required for the type of study being viewed should be included.

A remote station can be implemented using a standard nuclear medicine physician workstation, using a remote display of a nuclear medicine physician workstation, or using a remote viewing station (e.g., using a browser or with installation of remote viewing software) $(4,5)$.

\section{B. Data completeness}

All information needed for interpretation or consultation should be available to the physician at the remote location. This information includes demographic data, history, results of other relevant tests, procedure details, scintigraphic data, relevant correlative and structural imaging (as, for instance, CT and MR imaging data), technical problems, changes in the clinical condition of the patient, and indication for the study. All image data must be explicitly associated with patient identifier and appropriate label information.

\section{Data visualization}

The remote station should allow the same (or equivalent) display and processing functions as those used for interpretation or consultation at an on-site physician workstation. If the tele-nuclear medicine application involves a limited range of procedures, all functions needed to interpret or consult on those procedures should be provided.

Small displays (smart phones or tablets) may facilitate consultation with colleagues but will have limited utility for interpretation or reporting.

The following general abilities facilitate remote viewing: the ability to simultaneously display comparison studies with current and comparison data, adjust the size of the display viewport, pan and zoom, simultaneously show images of different sizes, and display image sequences in cine or montage format.

The following abilities provide control of the display intensity: the ability to display image data in gray scale or color and scale the intensity to fewer levels (e.g., 256 levels) for on-screen display, adjust upper and lower levels interactively for each dataset, determine the upper and lower pixel values used for scaling the display (this will help avoid scaling artifacts due to too few gray levels when data with a large dynamic range are reduced to fewer levels for display), choose from a set of color tables, apply lookup tables to adjust contrast or otherwise adjust contrast, and incorporate additional lookup tables.

The following abilities facilitate planar image display: the ability to display complete images ranging in size from $64 \times 64$ to $1,024 \times 1,024$ (including images that are not square and not powers of 2 ), display a $1,024 \times 1,024$ or $512 \times 1024$ whole-body image centered within a smaller-width frame (e.g., 256 pixels wide), intelligently trim zero or near-zero count regions from the periphery of the image to better use the screen area, simultaneously display whole-body images (e.g., 1,024 × 256) and spot images (e.g., $256 \times 256)$, and display a sequence of images scaled to a common maximum pixel value 
or scaled individually on the basis of the maximum pixel value in each image.

The following abilities facilitate dynamic image display: the ability to cine a dynamic sequence with up to a $256 \times 256$ matrix and 256 frames (scaled to a common maximum pixel value or scaled individually on the basis of the maximum pixel value in each image), reframe data (by combining images into fewer frames) at the time of display, and interactively change display thresholds and speed on each cine.

The following abilities facilitate gated planar image display: the ability to cine at least 3 views (8-32 frames, $64 \times 64$ to $128 \times 128$ matrix) at up to one full cardiac cycle per second, display views simultaneously and synchronously (preferably including the option for simple filtering; e.g., 9-point smooth), and display simultaneously at least 2 studies each with at least 3 views.

The following abilities facilitate tomographic image display (PET, SPECT, PET/CT, PET/MR imaging, and SPECT/CT): the ability to fuse separate anatomic and molecular imaging data; generate coronal, sagittal, or oblique images for display from a transaxial dataset; display multiple frames from a single axis, frames from 3 orthogonal axes, or an interactive multiaxis display; display, on the multiaxis display, at least 1-3 transaxial slices, 1-3 coronal slices, 1-3 sagittal slices, and 1 cine simultaneously; and navigate the multiaxis display, including clicking on any plane with automatic adjustment of the other 2 planes to that position, toggling on and off cursors on the multiaxis display to show the other image planes, and adjusting slice thickness at the time of display.

Display functions provided by myocardial analysis software packages enjoy considerable popularity. A remote station may provide this display functionality by running one of these packages or by displaying the processed screens (including designated cine screens) from these packages. Such display should include the ability to adjust upper and lower levels and to apply color lookup tables to the processed screens, as described above for other nuclear medicine images.

Intra- and intermodality registration may enable enhanced interpretation or consultation.

\section{Processing}

Minimal processing abilities should include the following: the ability to measure the value of a pixel or the average value from a region of interest, show the same quantitative data as are available on-site (e.g., maximum and mean standardized uptake value, normalizing for body weight or body surface area from a 2- or 3-dimensional region of interest), and smooth images (e.g., by a simple 9-point smooth) (recommended but not required).
Other processing may be included as necessary for a particular remote application, such as displaying an activity profile, performing renal analysis, calculating gallbladder emptying, or calculating gastric emptying.

\section{E. Communications}

The communications protocol should allow for confirmation of reliable transmission. Encrypted transmission of data will improve the security of transmission over public channels. Many current communication technologies provide adequate speed for most tele-nuclear medicine applications. In the oncall setting, correlative imaging requirements will generally dominate selection of a communication speed.

\section{F. Compression}

Data can be compressed to improve the speed of data transmission, although speed of transmission may not be an issue for nuclear medicine data alone. If structural data are included, speed is an issue.

Compression can be either lossless, where the uncompressed data are identical to the original, or lossy, where the uncompressed data differ from the original. If lossy compression is used, the remote data should be diagnostically comparable to the original.

\section{G. Monitor quality control $(6,7)$}

\section{Considerations}

The remote location may present special consideration for the quality control of the monitor on which nuclear medicine information is displayed. The remote monitor should undergo the same quality control procedures as on-site monitors (see SNMMI Guideline for General Imaging). Remote monitors, particularly for the on-call application, may have multiple uses, and the monitor setup may be altered by non-nuclear medicine applications. Alterations to the color depth of the display (e.g., 256 colors vs. millions of colors) may significantly affect image interpretation yet not be immediately apparent on casual inspection of image data. 2. Test patterns

A test pattern such as the Society of Motion Picture and Television Engineers (SMPTE) medical diagnostic imaging test pattern may be used to check the spatial resolution and linearity of a monitor. For the SMPTE pattern, the lines should appear linear and all line pairs of different widths should be visible and have the same contrast (8). A test pattern such as that of the SMPTE may be used to check gray-scale linearity. For the SMPTE, the 5\% and $95 \%$ boxes should be visible, and the change between intensity levels should appear linear. A test pattern such as that of the Brigham and Women's Hospital can be used to 
check for discontinuities in the gray scale, which can produce artificial edges in image data. For the Brigham and Women's Hospital test pattern, the change in intensity should be continuous without any visible rings (http://parker.bidmc.harvard.edu/TestPatterns. html). The remote monitor should be checked visually for any gross color dysfunction (e.g., absence of one of the RGB signals). If a locally installed color scale is used for remote viewing, it should be verified as being visually similar to that installed at the primary site, with color transitions at the same locations.

3. Quality control procedures

Full quality control of a monitor (7) is not practical for remote sites; however, relatively simple test patterns should be readily displayable. There should be a regular protocol and schedule of quality control testing. In some multiuse settings, quality control may need to be tested for each tele-nuclear medicine session. Tele-nuclear medicine systems that show test patterns at the time of login facilitate regular quality control.

\section{H. Security}

Data on nuclear medicine procedures, including the fact that such a procedure was performed, are confidential medical information. The goal of security is to decrease the probability of unauthorized access while impeding authorized access as little as possible. The benefit from increased security should be balanced against costs, including the cost of decreased availability of information to authorized users. Electronic transmission should be made more secure for nuclear medicine data than for traditional nondigital hospital practices. Access should be limited to only authorized individuals both in transit and at the tele-nuclear medicine site. Security includes login, communications, and access to data stored on the remote system. A time-out period that is appropriate for the environment in which the remote workstation is being used may be implemented. Nuclear medicine is expected to be a low-priority target, and the image portion of the data is meaningful to a limited audience. Thus, it may be appropriate to place greater emphasis on securing system login procedures and access to medical databases containing patient information rather than on encrypting pure image data. There should be a disaster recovery plan dealing with breach of system security or loss of source data due to malfunction of the equipment.

\section{Issues Requiring Further Clarification}

The extent to which lossy compression provides diagnostically equivalent information when used either for primary interpretation or for correlative images needs further clarification. Legal issues about remote interpretation are still evolving.

\section{DOCUMENTATION/REPORTING}

See the SNMMI Guideline for General Imaging.

\section{EQUIPMENT SPECIFICATION}

Equipment specifications for each procedure are given in the respective practice guidelines.

\section{QUALITY CONTROL AND IMPROVEMENT, SAFETY, INFECTION CONTROL, AND PATIENT EDUCATION CONCERNS}

See also the SNMMI Guideline for General Imaging.

Policies and procedures related to quality, patient education, infection control, and safety should be developed and implemented in accordance with the ACR policy on quality control and with patient education concerns appearing elsewhere in the ACR practice guidelines and technical standards.

Physician quality control should also be done regularly to ensure that physicians interpret results consistently and accurately. The ACR Radpeer system offers one method of achieving this goal; however, Radpeer is not mandatory and equivalent systems may be used.

The performance of equipment should be monitored in accordance with ACR technical standards for medical nuclear physics performance monitoring of CT and nuclear medicine equipment, as well as according to the EANM guidelines on routine quality control (9) and acceptance testing (10) for nuclear medicine instrumentation.

Information specific to the procedure should also be included in each guideline.

\section{RADIATION SAFETY IN IMAGING}

See the appropriate study guideline.

\section{ACKNOWLEDGMENTS}

The Committee on SNMMI Guidelines consists of the following individuals: Kevin J. Donohoe, MD (Chair) (Beth Israel Deaconess Medical Center, Boston, MA); Sue Abreu, MD (Sue Abreu Consulting, Nichols Hills, OK); Helena Balon, MD (Beaumont Health System, Royal Oak, MI); Twyla Bartel, DO (UAMS, Little Rock, AR); Paul E. Christian, CNMT, BS, PET (Huntsman Cancer Institute, University of Utah, Salt Lake City, UT); Dominique Delbeke, MD (Vanderbilt University Medical Center, Nashville, TN); Vasken Dilsizian, MD (University of Maryland Medical Center, Baltimore, MD); Kent Friedman, MD (NYU School of Medicine, New York, NY); James R. Galt, PhD (Emory University Hospital, Atlanta, GA); Jay A. Harolds, MD (OUHSC, Department of Radiological Science, Edmond, OK); Aaron Jessop, MD (UT MD Anderson Cancer Center, Houston, TX); David H. Lewis, MD (Harborview Medical Center, Seattle, WA); J. Anthony Parker, MD, $\mathrm{PhD}$ (Beth Israel Deaconess Medical Center, Boston, MA); James A. Ponto, RPh, BCNP (University of Iowa, Iowa City, IA); 
Lynne T. Roy, CNMT (Cedars-Sinai Medical Center, Los Angeles, CA); Heiko Schoder, MD (Memorial SloanKettering Cancer Center, New York, NY); Barry L. Shulkin, MD, MBA (St. Jude Children's Research Hospital, Memphis, TN); Michael G. Stabin, PhD (Vanderbilt University, Nashville, TN); and Mark Tulchinsky, MD (Milton S. Hershey Medical Center, Hershey, PA).

The EANM Board consists of the following individuals: Fred Verzijlbergen, MD, PhD (Erasmus MC Central Location, Rotterdam, The Netherlands); Arturo Chiti, MD (Istituto Clinico Humanitas, Rozzano Milan, Italy); Savvas Frangos, MD (Bank of Cyprus Oncology Center, Strovolos, Nicosia, Cyprus); Jure Fettich, MD (University Medical Centre Ljubljana, Ljubljana, Slovenia); Bernd J. Krause, MD, PhD (Universitätsklinikum Rostock, Rostock, Germany); Dominique Le Guludec, PhD (Hopital Bichat, Paris, France); and Wim Oyen, MD, PhD (Radboud University Medical Centre, Nijmegen, The Netherlands).

\section{REFERENCES}

1. The DICOM standard. Digital Imaging and Communications in Medicine Web site. http://medical.nema.org/standard.html. Accessed October 10, 2013

2. Benjamin M, Aradi Y, Shreiber R. From shared data to sharing workflow: merging PACS and teleradiology. Eur J Radiol. 2010;73:3-9.
3. Silva E III, Breslau J, Barr RM, et al. ACR white paper on teleradiology practice: a report from the Task Force on Teleradiology Proactive. J Am Coll Radiol 2013;10:575-585.

4. Slomka PJ, Elliott E, Driedger AA. Java-based remote viewing and processing of nuclear medicine images: toward "the imaging department without walls." J Nucl Med. 2000;41:111-118.

5. Wallis JW. Java and teleradiology. J Nucl Med. 2000;41:119-122.

6. Nawfel RD, Chan KH, Wagenaar DJ, Judy PF. Evaluation of video gray-scale display. Med Phys. 1992;19:561-567.

7. Samei E, Badano A, Chakraborty D, et al. Assessment of display performance for medical imaging systems. http://deckard.mc.duke.edu/ samei/tg18. Carl E. Ravin Advanced Imaging Laboratories Web site. Updated January 10, 2006. Accessed October 10, 2013.

8. Gray JE, Lisk KG, Anderson W, et al. Acceptance and use of the SMPTE medical diagnostic imaging test pattern for television monitors and hard-copy recording cameras. SMPTE Mot Imaging J. 1990;99:1001-1007.

9. Busemann Sokole E, Plachcinska A, Britten A, et al. Routine quality control recommendations for nuclear medicine instrumentation. Eur J Nucl Med Mol Imaging. 2010;37:662-671.

10. Busemann Sokole E, Plachcinska A, Britten A, et al. Acceptance testing for nuclear medicine instrumentation. Eur $J$ Nucl Med Mol Imaging. 2010;37:672-681.

\section{APPROVAL DATES}

This practice guideline (version 2.0) was approved by the Board of Directors of the SNMMI on November 25, 2013, and by the EANM Board on November 10, 2013. Version 1.0 was approved on June 15, 2002. 